University of Nebraska - Lincoln

DigitalCommons@University of Nebraska - Lincoln

2010

\title{
Contributions of demography and dispersal parameters to the spatial spread of a stage-structured insect invasion
}

Tom E. X. Miller

Rice University, tom.miller@rice.edu

Brigitte Tenhumberg

University of Nebraska - Lincoln, btenhumberg2@unl.edu

Follow this and additional works at: https://digitalcommons.unl.edu/bioscifacpub

Part of the Life Sciences Commons

Miller, Tom E. X. and Tenhumberg, Brigitte, "Contributions of demography and dispersal parameters to the spatial spread of a stage-structured insect invasion" (2010). Faculty Publications in the Biological Sciences. 95.

https://digitalcommons.unl.edu/bioscifacpub/95

This Article is brought to you for free and open access by the Papers in the Biological Sciences at DigitalCommons@University of Nebraska - Lincoln. It has been accepted for inclusion in Faculty Publications in the Biological Sciences by an authorized administrator of DigitalCommons@University of Nebraska - Lincoln. 


\title{
Contributions of demography and dispersal parameters to the spatial spread of a stage-structured insect invasion
}

\author{
Tom E. X. Miller ${ }^{1,3}$ And Brigitte Tenhumberg ${ }^{2}$ \\ ${ }^{1}$ Department of Biological Science, Florida State University, Tallahassee, Florida 32306 USA \\ ${ }^{2}$ School of Biological Sciences and Department of Mathematics, University of Nebraska, Lincoln, Nebraska 68588 USA
}

\begin{abstract}
Stage-structured models that integrate demography and dispersal can be used to identify points in the life cycle with large effects on rates of population spatial spread, information that is vital in the development of containment strategies for invasive species. Current challenges in the application of these tools include: (1) accounting for large uncertainty in model parameters, which may violate assumptions of "local" perturbation metrics such as sensitivities and elasticities, and (2) forecasting not only asymptotic rates of spatial spread, as is usually done, but also transient spatial dynamics in the early stages of invasion. We developed an invasion model for the Diaprepes root weevil (DRW; Diaprepes abbreviatus [Coleoptera: Curculionidae]), a generalist herbivore that has invaded citrusgrowing regions of the United States. We synthesized data on DRW demography and dispersal and generated predictions for asymptotic and transient peak invasion speeds, accounting for parameter uncertainty. We quantified the contributions of each parameter toward invasion speed using a "global" perturbation analysis, and we contrasted parameter contributions during the transient and asymptotic phases. We found that the asymptotic invasion speed was $0.02-0.028 \mathrm{~km} /$ week, although the transient peak invasion speed $(0.03-$ $0.045 \mathrm{~km} /$ week) was significantly greater. Both asymptotic and transient invasions speeds were most responsive to weevil dispersal distances. However, demographic parameters that had large effects on asymptotic speed (e.g., survival of early-instar larvae) had little effect on transient speed. Comparison of the global analysis with lower-level elasticities indicated that local perturbation analysis would have generated unreliable predictions for the responsiveness of invasion speed to underlying parameters. Observed range expansion in southern Florida (1992-2006) was significantly lower than the invasion speed predicted by the model. Possible causes of this mismatch include overestimation of dispersal distances, demographic rates, and spatiotemporal variation in parameter values. This study demonstrates that, when parameter uncertainty is large, as is often the case, global perturbation analyses are needed to identify which points in the life cycle should be targets of management. Our results also suggest that effective strategies for reducing spread during the asymptotic phase may have little effect during the transient phase.
\end{abstract}

Key words: Diaprepes abbreviatus; Florida citrus pest; generalist herbivore; integro-difference model; invasive insect; parameter uncertainty; population dynamics; root weevil; sensitivity analysis; spatial spread; transient dynamics.

\section{INTRODUCTION}

Biological invasions by exotic species occur in three phases: arrival, establishment, and spread (Elton 1958). It is generally during the third phase, spatial spread, that exotic species pose the greatest threats to agriculture, natural resources, and native species (National Research Council 2002). The ability to forecast when and where nonindigenous species will spread could increase the likelihood that invasions are detected at incipient stages, when eradication attempts are most successful (Fagan et al. 2002), and could contribute to the development of

Manuscript received 12 March 2009; revised 15 July 2009; accepted 27 July 2009. Corresponding Editor: M. P. Ayres.

3 Present address: Department of Ecology and Evolutionary Biology, Rice University, Houston, Texas 77005 USA. E-mail: tom.miller@rice.edu proactive management strategies (Sharov and Liebhold 1998). Thus, there is need for accurate predictions of invasive spread following the arrival and establishment of exotic species, and implementation of management strategies informed by such predictions.

Mathematical models of spatial population dynamics can contribute to the prediction and management of range expansion by invasive species in at least two ways. First, spatial models can be used to estimate the speed of population expansion (linear distance/time) based on relatively few parameters that describe demographic rates and dispersal abilities (Kot et al. 1996, Neubert and Caswell 2000, Okubo and Levin 2002). Invasion speeds have been estimated in this way for a diverse array of invasive organisms (e.g., Andow et al. 1990, Hastings et al. 2005), although the consistency of predicted and observed rates of spread is mixed (Grosholz 1996). 
Second, for invaders with stage-structured demography and dispersal (including many plants, vertebrates, and insects), perturbation analyses of stage-structured spatial models can be used to identify which features of the life cycle make the greatest contributions to the speed of invasion (Neubert and Caswell 2000). Insights from such analyses are useful for developing management strategies, targeted at sensitive points in the life cycle, to slow or halt the spread of invaders (Buckley et al. 2005) or accelerate the spread of recovering species (Tinker et al. 2008). In addition, perturbation analyses may suggest particular parameter estimates for which more or better empirical data are needed.

Despite recent advances, there are at least two key challenges in our abilities to predict, understand, and manage spatial spread, particularly for stage-structured invasions. First, there is often a large amount of uncertainty in the parameter estimates on which invasion models are based. Although parameter uncertainty can complicate any attempt to integrate theory and application, it is especially problematic for invasion models because dispersal parameters are both notoriously difficult to measure (Nathan et al. 2003) and critically important determinants of invasion speed (Kot et al. 1996). Thus, there is a need for quantitative approaches that can help to guide policy makers and resource managers in the formulation of effective control strategies in spite of this uncertainty (Buckley et al. 2005). Sensitivities and elasticities (types of perturbation analysis that provide rankings of parameter importance) are widely used in applied ecology for exactly this purpose (Caswell 2001). However, because these methods provide a strictly "local" estimate of a parameter's importance (i.e., slope of the response function at or very near the parameter's value), their application is only appropriate when examining the consequences of very small perturbations to very wellknown parameters (Horvitz and Schemske 1995, Caswell 2001). When parameter uncertainty is large, as is often the case, alternative, "global" methods of perturbation analysis are required. Such methods have been used to analyze models of temporal invasion dynamics (Tenhumberg et al. 2008) but have not yet been applied to models of spatial spread.

A second challenge is the inclusion of nonequilibrium dynamics in model predictions and management approaches. There is growing interest in transient population dynamics in applied ecology and invasion biology (Koons et al. 2005, Townley et al. 2007, McMahon and Metcalf 2008, Tenhumberg et al. 2009), but few studies have considered transient spatial spread dynamics (Caswell 2007). Rather, most analyses of spatial spread focus on long-term, asymptotic predictions, when the advancing "wave" of organisms has reached a constant shape and a stable distribution of individuals among life stages. Real invasions, by contrast, often begin at a single location with the human-mediated transport of a few individuals representing a single life stage, such as
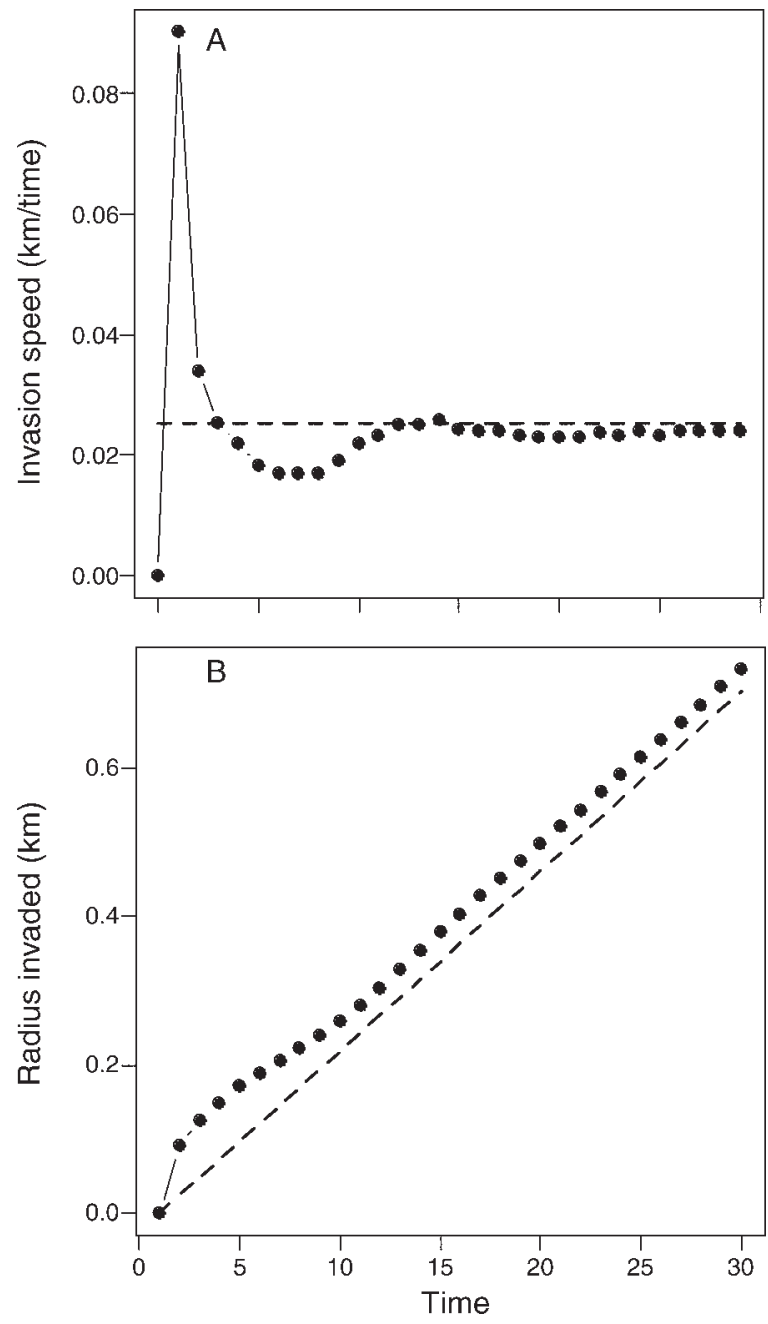

Fig. 1. (A) Example of transient spatial spread dynamics. After an initial overshoot (the transient peak), the invasion speed converges on its predicted asymptotic value (dotted line). (B) Transient dynamics shown in panel (A) have lasting effects on range expansion. Dots show the cumulative radius invaded through time, including transient dynamics, and the dashed line shows the expectation based solely on the asymptotic invasion speed. Time here is arbitrary and unitless.

plant seeds or adult insects. Such deviation from asymptotic conditions can cause an invasive population to initially "overshoot" its long-term rate of spread (Fig. 1A). Transient peaks in invasion speed result in an initial burst of spread, providing a "head start" that may have lasting effects on patterns of range expansion, even when asymptotic conditions are quickly reached (Fig. 1B). Management practices that depress transient peaks in invasion speed may therefore aid in long-term containment. Yet, no previous studies have examined whether management techniques aimed to depress transient peaks in invasion speed should differ from those aimed to decelerate asymptotic speed.

With these issues in mind, we developed a stagestructured model for the spatial spread of an invasive 
exotic insect, the Diaprepes root weevil (DRW; Diaprepes abbreviatus). This generalist herbivore is native to Caribbean islands and was first introduced to central Florida, USA in the 1960s on a shipment of ornamental plants from Puerto Rico (Woodruff 1968). The DRW now occurs throughout central and southern Florida, where it is an economically important citrus pest; the weevil's high potential for human-mediated, long-distance transport also threatens agricultural, ornamental, and native plants elsewhere in the United States (Grafton-Cardwell et al. 2004). We used this insect invasion case study to address current challenges, relating to parameter uncertainty and transient dynamics, in our ability to forecast and manage spatial spread of stagestructured invasions. Invasive spread by insects with nonoverlapping generations has been analyzed using unstructured invasion models, in which the densities and locations of a single stage (usually adults) are tracked from one time step to the next (e.g., Andow et al. 1990, Johnson et al. 2006). However, the DRW has long-lived larval and adults stages, has overlapping generations, and shows persistent stage structure in the warm climates of its native and introduced ranges (Beavers and Selhime 1976). Therefore, stage-structured invasion models are an appropriate tool for analyzing this species' spread dynamics, and our approach is applicable to other invasive insects with similar life histories.

The DRW invasion provides a valuable and relevant case study of stage-structured invasion dynamics for a number of reasons. First, the weevil has been discovered recently in the Rio Grande Valley of Texas (Skaria and French 2001) and in urban areas of Los Angeles and San Diego counties, California (Lapointe et al. 2007). Clearly, there is high risk of human-mediated introductions of DRW to citrus-growing areas outside of Florida (GraftonCardwell et al. 2004). Knowledge of the mechanisms underlying transient and asymptotic rates of spread could aid in the management of established, emerging, and future DRW invasions. Natural enemies that attack egg, larval, and adult stages have been explored as biocontrol agents (Grafton-Cardwell et al. 2004), yet demographic analyses to guide such practices are currently lacking. Second, due to its economic importance, demography and dispersal data are available for model construction, although there are gaps (and therefore uncertainty) in the data set. Most of the existing data were collected under laboratory conditions, so there is an additional element of uncertainty regarding how laboratory-based parameter estimates translate to the field. Third, DRW spread in Florida has been carefully documented, providing independent data with which to evaluate how closely model predictions match observed rates of spatial spread.

This study had three specific objectives. First, we estimated and contrasted asymptotic and transient invasion speed based on existing demography and dispersal data. Second, we estimated and contrasted the contributions of the underlying demographic and dispersal parameters to transient and asymptotic inva- sion speed. Because there was uncertainty in the available data, we estimated invasion speeds and parameter contributions by sampling parameters from uncertainty ranges. Finally, we compared predicted rates of DRW invasion with independent data on invasive range expansion in Florida, USA.

\section{Methods \\ Focal species}

The Diaprepes root weevil (DRW) is a long-lived, holometabolous, multivoltine insect (Coleoptera: Curculionidae). Females deposit egg clusters on the leaves of host plants. Although the host range includes more than 270 species from 59 families (Simpson et al. 1996), the DRW has received most attention as a pest of citrus. Newly hatched larvae drop to the soil surface and burrow underground where they feed on roots. Larvae develop through 10-11 instars over a period of 5-7 months. Upon adult eclosion, there is a pre-reproductive period before adults reach sexual maturity (Beavers 1982). Adults can live for more than five months and females can lay $>6000$ eggs over their lifetimes. Adult weevils are capable fliers but move relatively short distances (Nigg et al. 2001). In southern Florida and in its native Puerto Rico, the weevil has overlapping generations and shows persistent stage structure, with adults occurring year-round (Beavers and Selhime 1976).

The weevil was first discovered in Apopka, Florida in 1964. The threat to citrus growers prompted weevil eradication efforts during the 1960s and 1970s, but these efforts were dropped due to ineffective insecticides and negative effects on pollinators (Hall 2001). The weevil subsequently spread throughout peninsular Florida. Human movement of infested plant material is thought to have played a major role in its statewide spread dynamics (Bas et al. 2000). Current DRW management practices in Florida include biological control targeted at eggs (parasitic wasps) and larvae (entomopathogenic nematodes); entomopathogenic fungi that target adults are also being explored (Grafton-Cardwell et al. 2004). Recently, long-distance movement of infested plant material from Florida has led to weevil establishment in California and Texas, USA (Grafton-Cardwell et al. 2004).

\section{Model development}

Our theoretical approach is based on the integrodifference invasion model developed by Kot et al. (1996) and extended to stage-structured populations by Neubert and Caswell (2000). The model tracks the population stage vector $\mathbf{n}$, which holds the densities of each demographic class or life stage, through discrete time $(t)$ and continuous space $(x)$ according to an integro-difference equation that combines demography and dispersal:

$$
\mathbf{n}(x, t+1)=\int_{-\infty}^{\infty}\left[\mathbf{K}(x-y) \circ \mathbf{A}_{\mathbf{n}}\right] \mathbf{n}(y, t) d y .
$$


The population projection matrix $\mathbf{A}_{\mathbf{n}}$ contains stasis probabilities (on the diagonal), progression probabilities (on the sub-diagonal), and fecundities (on the top row). The subscript $\mathbf{n}$ denotes that the elements of $\mathbf{A}$ may be density dependent. The matrix $\mathbf{K}$ contains dispersal kernels (probability distributions of dispersal distances) corresponding to each element of $\mathbf{A}$. The $i j$ th element of $\mathbf{K}$ gives the probability that an individual moves distance $(x-y)$ during the transition from stage $i$ to stage $j$. Thus, the population vector at location $x$ and time $t+1$ represents all the propagules produced at location $y$ and time $t$ that dispersed distance $(x-y)$, integrated over all $y$. Neubert and Caswell (2000) show that the asymptotic speed $\left(c^{*}\right)$ of a population front advancing through homogenous space can be estimated directly from the demography (A) and dispersal (K) matrices.

The DRW invasion model includes six weevil life stages: eggs (E); early-instar, neonate (less than $\sim 2$ week-old) larvae (N); later-instar larvae (L); pupae (P); juvenile, pre-reproductive adults $(\mathrm{J})$; and reproductively mature adults (A). Our model considers only females; there is no evidence for sex bias in dispersal behavior (Nigg et al. 2001). We separated larvae and adults each into two stages because the empirical literature suggested that these distinctions were biologically meaningful (Table 1). For example, Lapointe (2000) reported mortality differences for early-instar $(<2$ week-old) and later-instar larvae, and Beavers (1982) reported a lag between adult eclosion and oviposition.

The elements of the population projection matrix are composed of the lower-level parameters $\sigma_{i}$ (probability that an individual of stage $i$ survives over one time step), $\gamma_{i}$ (probability that an individual of stage $i$ graduates over one time step), and $\phi$ (female eggs per mature female per time step). We estimated $\gamma_{i}$ according to the geometric distribution method (Caswell 2001), where the probability of graduating from stage $i$ is equal to the inverse of stage $i$ development time, $\gamma_{i}=\tau_{i}^{-1}$. Additionally, because the mature adult stage is terminal, we estimated the adult stasis element $\left(\mathbf{A}_{6,6}\right)$ as 0.5 raised to the inverse of adult life span. That is, we assumed that $50 \%$ of adults survive until the mean life span. Finally, because DRW reproduction is continuous over the adult life span, we calculated fertility as a birth-flow process (Caswell 2001:171). The projection matrix is therefore:

$$
\mathbf{A}=\left[\begin{array}{ccc}
\left(1-\tau_{\mathrm{E}}^{-1}\right) \sigma_{\mathrm{E}} & 0 & 0 \\
\tau_{\mathrm{E}}^{-1} \sigma_{\mathrm{E}} & \left(1-\tau_{\mathrm{N}}^{-1}\right) \sigma_{\mathrm{N}} & 0 \\
0 & \tau_{\mathrm{N}}^{-1} \sigma_{\mathrm{N}} & \left(1-\tau_{\mathrm{L}}^{-1}\right) \sigma_{\mathrm{L}} \\
0 & 0 & \tau_{\mathrm{L}}^{-1} \sigma_{\mathrm{L}} \\
0 & 0 & 0 \\
0 & 0 & 0
\end{array}\right.
$$

To incorporate density dependence in the projection matrix (Eq. 1), we multiplied all matrix elements by a Ricker-type density penalty, $e^{-b N}$, where the parameter $b$ determines the strength of density dependence and $N$ is the total population size (Ricker 1954). The introduction of density dependence results in a local equilibrium population size, which was convenient for estimating the velocity of the traveling wave. However, the value of this parameter had no effect on invasion speed, as expected (Kot et al. 1996), and so we set $b=$ 0.0001 and do not consider this parameter further.

Only the pre-reproductive and mature adults are capable of autonomous dispersal (we ignore small-scale larval movement). Therefore, the matrix $\mathbf{K}(x-y)$ contains pre-reproductive and mature adult dispersal kernels, $k_{\mathrm{J}}(x-y)$ and $k_{\mathrm{A}}(x-y)$, at only those entries involving transitions of these stages, and delta functions, $\delta(x-y)$, everywhere else (Neubert and Caswell 2000). Delta functions have probability 1 for $x=y$ and probability zero for $x \neq y$. For dispersing stages, the model is flexible with regard to the particular form of the dispersal kernel, although analytical wave speed solutions require that the kernel have moments (Kot et al. 1996).

\section{Parameter estimation}

We used data available in the literature to estimate parameter ranges for the DRW demography (A) and dispersal (K) matrices (Table 1). Demographic data consisted of stage durations and cohort survival estimates from laboratory studies of the DRW life cycle. In some studies, experiments were conducted at multiple temperatures. In these cases, we used data from $22^{\circ} \mathrm{C}$; this temperature was similar to conditions from other studies that did not vary temperature and close to the mean annual temperature in the weevil's invasive range $\left(22.9^{\circ} \mathrm{C}\right.$; $2003-2005$ data from Belle Glade, Ft. Lauderdale, Homestead, and Immokalee, Florida, USA). For each parameter, we estimated the uncertainty range as the $95 \%$ confidence interval (CI) derived from means, sample sizes, and variances. We assumed that development time data were normally distributed and that survival data were binomially distributed. For some parameters (pre-reproductive adult survival), no data were available, so we based uncertainty ranges on qualitative suggestions in the literature (Table 1). Although we define the early-instar larval stage based on age ( $\leq 2$ weeks), we included an uncertainty range on

$\left.\begin{array}{ccc}0 & \sqrt{\sigma_{\mathrm{E}}} \frac{\phi \tau_{\mathrm{J}}^{-1} \sigma_{\mathrm{J}}}{2} & \sqrt{\sigma_{\mathrm{E}}} \frac{\phi+\phi 0.5^{1 / \tau_{\mathrm{A}}}}{2} \\ 0 & 0 & 0 \\ 0 & 0 & 0 \\ \left(1-\tau_{\mathrm{P}}^{-1}\right) \sigma_{\mathrm{P}} & 0 & 0 \\ \tau_{\mathrm{P}}^{-1} \sigma_{\mathrm{P}} & \left(1-\tau_{\mathrm{J}}^{-1}\right) \sigma_{\mathrm{J}} & 0 \\ 0 & \tau_{\mathrm{J}}^{-1} \sigma_{\mathrm{J}} & 0.5^{1 / \tau_{\mathrm{A}}}\end{array}\right]$.


TABLE 1. Demography and dispersal parameters, parameter uncertainty ranges (minimummaximum), and data sources for the Diaprepes root weevil (Diaprepes abbreviatus) invasion model.

\begin{tabular}{clcl}
\hline \hline Parameter & \multicolumn{1}{c}{ Description } & Uncertainty range & \multicolumn{1}{c}{ Source } \\
\hline$\tau_{\mathrm{E}}$ & Egg development time (weeks) & $1.39-1.76$ & Lapointe (2001) \\
$\sigma_{\mathrm{E}}$ & Weekly egg survival & $0.79-0.88$ & Lapointe (2001) \\
$\tau_{\mathrm{N}}$ & Early-instar larva development time (weeks) $\dagger$ & $1.71-2.29$ & \\
$\sigma_{\mathrm{N}}$ & Weekly early-instar larva survival & $0.77-0.95$ & Lapointe (2000) \\
$\tau_{\mathrm{L}}$ & Late-instar larva development time (weeks) & $21.04-25.88$ & Lapointe (2000) \\
$\sigma_{\mathrm{L}}$ & Weekly late-instar larva survival & $0.98-0.99$ & Lapointe (2000) \\
$\tau_{\mathrm{P}}$ & Pupa development time (weeks) & $4.49-5.22$ & Lapointe (2000) \\
$\sigma_{\mathrm{P}}$ & Weekly pupa survival & $0.97-0.99$ & Lapointe (2000) \\
$\tau_{\mathrm{J}}$ & Pre-reproductive period of adults (weeks) & $2.22-4.01$ & Beavers (1982) \\
$\sigma_{\mathrm{J}}$ & Pre-reproductive adult survivalł & $0.95-1.0$ & \\
$\tau_{\mathrm{A}}$ & Mature adult lifespan (weeks) & $13.98-21.78$ & Beavers (1982) \\
$\phi$ & Mature adult fecundity (eggs/week) & $167.81-191.5$ & Beavers (1982) \\
$\alpha_{\mathrm{J}}$ & Pre-reproductive adult dispersal (km/week) & $0.023-0.032$ & Nigg et al. (2001) \\
$\alpha_{\mathrm{A}}$ & Mature adult dispersal (km/week) & $0.023-0.032$ & Nigg et al. (2001) \\
\hline
\end{tabular}

$\dagger$ Uncertainty range corresponds to the 2-week period during which mortality was estimated (Lapointe 2000).

$\ddagger$ Beavers (1982) suggests high survival of pre-reproductive adults. The weekly survival range corresponds to a cohort survival rate of $0.9-1.0$.

early-instar development time $\left(\tau_{N}\right)$ to examine the response of invasion speed to variation in this interval of elevated larval mortality (Table 1). We set all development times to units of weeks and scaled the survival estimates to represent weekly survival; this ensured that demographic transitions occurred on the same timescale as dispersal. Thus, the time step of the model is one week.

DRW dispersal data came from a field experiment conducted by Nigg et al. (2001). These investigators marked, released, and resighted adult weevils in a citrus grove in Lake County, Florida at weekly intervals over a 10 -week period. In total, 580 weevils were marked and released and 146 were resighted within a $120 \mathrm{~m}$ radius from the release point, the farthest distance searched. These dispersal data are shown in Fig. 2. We fit a LaPlace dispersal kernel $\left(K=e^{-|x| / \alpha} / 2 \alpha\right)$ to the DRW dispersal data using maximum likelihood methods (Burnham and Anderson 2002) and used the likelihood profile method (Hilborn and Mangel 1997) to generate a 95\% CI for the LaPlace parameter $\alpha$, which is the mean dispersal distance. The LaPlace distribution provided a good fit to the dispersal data of Nigg et al. (2001), much better than a normal distribution $(\triangle \mathrm{AIC}=15.4)$. The reproductive status of the weevils used in the dispersal experiment was not determined; thus separate dispersal estimates for pre-reproductive and mature adults were not available. We therefore used the $95 \%$ CI on $\alpha$ as the uncertainty range for both pre-reproductive $\left(\alpha_{\mathrm{J}}\right)$ and mature $\left(\alpha_{\mathrm{A}}\right)$ adults (Table 1$)$.

\section{Monte Carlo-based perturbation analyses of asymptotic and transient dynamics}

We constructed a spatially explicit, stage-structured simulation model of DRW invasion dynamics (Eq. 1) using the programming language $C$. Simulated invasions occurred within a linear array of cells, where each cell represented a $0.001-\mathrm{km}$ spatial interval. At each time step, populations within individual cells increased according to the local population projection matrix (A). Then individuals were redistributed throughout the array from their "source" cell according to distance- and life-stage-dependent probabilities given by the dispersal matrix $(\mathbf{K})$. We used numerical integration to estimate the area under the dispersal kernel corresponding to the distance between "source" and "target" cells. The combination of local population growth and spatial redistribution of propagules generated a traveling wave of simulated weevils.

To evaluate the effect of parameter uncertainty on predicted invasion speed, we conducted a Monte Carlo

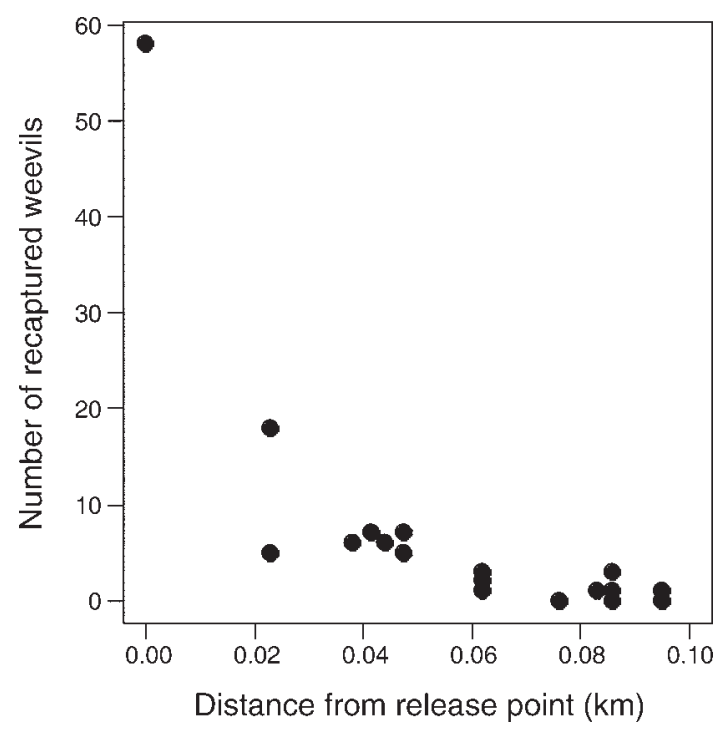

FIG. 2. Results of mark-release experiment used to fit the DRW (Diaprepes root weevil, Diaprepes abbreviatus) dispersal kernels. Data are from Nigg et al. (2001). 
perturbation analysis and calculated partial rank correlation coefficients (PRCC) to describe the statistical effect of each input parameter on model output (Tenhumberg et al. 2008). We constructed a Latin Hypercube (Blower and Dowlatabadi 1994) by dividing the range of each of our 14 parameter estimates (Table 1) into 1000 equi-probable intervals using uniform distributions; the midpoint of each interval was used for the Latin Hypercube. The Latin Hypercube creates 1000 unique parameter combinations by sampling without replacement from the set of equi-probable intervals. The procedure assured that extreme values were included in the perturbation analysis, which minimizes the number of required simulations (1000 simulations took 30 days). As a rule of thumb, Blower and Dowlatabadi (1994) suggest a minimum of $0.75 \mathrm{~K}$ iterations, where $K$ is the number of uncertain parameters. In our case, $K=14$, so our number of simulations was two orders of magnitude larger than the minimum. Our perturbation analysis was based on uniform distributions because we lacked information on the true parameter distributions and previous work has shown that the choice of distribution has little effect on the qualitative conclusions of the analysis (Tenhumberg et al. 2008).

Estimation of wave speed from numerical simulation requires a density threshold for determining the location of the invasion front; we set the threshold to $5 \%$ of the local equilibrium density. For each iteration, we estimated asymptotic speed as the rate of spread (distance/time) during the final time step. Thus, we assumed that the duration of the simulation (40 weeks) was sufficient to reach asymptotic conditions. We evaluated the validity of this assumption by comparing values of asymptotic speed from the simulations with the analytical wave speed solution (Neubert and Caswell 2000: Eq. 24), calculated for each unique parameter combination.

We estimated transient peak invasion speed as the maximum rate of spread over the duration of the simulation; the transient peak always occurred during the first few time steps (as in Fig. 1). Because the magnitude of transient dynamics can be strongly dependent upon initial stage structure (e.g., McMahon and Metcalf 2008), it is important to consider realistic initial conditions of invasion. Although all DRW invasions in North America are thought to have originated from movement of infested plant material, it is not known what stage or combination of stages made up the initial cohort of invaders. We chose an initial distribution consisting entirely of mature adults. Because they are long-lived and feed within plant canopies, adult weevils have high potential for effective, human-mediated, long-distance transport on ornamental or agricultural plants. Thus, a small cohort of adults is one likely scenario for the initial conditions of DRW invasions, although other scenarios are also possible (e.g., transport of larvae in soil). We estimated from the simulated invasions the mean and 95\% confidence intervals for the asymptotic and transient invasion speeds, which incorporate uncertainty in the input parameters.

We analyzed the statistical relationships between each parameter and each response variable (asymptotic or transient peak invasion speed) using partial rank correlation coefficients (PRCC). This nonparametric approach was necessary because parameter values were not normally distributed and because response variables were not necessarily linear functions of input variables. PRCC values range from -1 to +1 and their absolute values do not sum to 1 . The PRCCs provided estimates of the relative importance of individual demography and dispersal parameters (Blower and Dowlatabadi 1994, Tenhumberg et al. 2008), even with unknown levels of correlation among parameters (Conover 1980). For comparison with the more traditional approach, we also estimated the lower-level elasticities (LLE) of the demography and dispersal parameters using the formulae in the Appendix, which are based on the analytical methods described in Buckley et al. (2005). LLEs are local estimates of parameter importance in that they provide the slope of the response function (wave speed, in this case) at or very near a parameter's value. Unlike the Monte Carlo-PRCC approach, LLE analysis assumes that the effect of a parameter perturbation is independent of other parameter values, and that perturbations to parameters values are infinitesimally small (extrapolation to the effect of larger perturbations requires a linear response function). We restrict the comparison of "global" (PRCC) vs. "local" (LLE) analyses to asymptotic invasion speed. We evaluated LLEs at the midpoints of the parameter ranges.

\section{Observed DRW spread dynamics}

Finally, we compared predicted rates of range expansion with the observed spread dynamics of the DRW in southern Florida. We focus on this region because our model assumes temporally constant parameter values; demographic parameters are likely to be influenced by seasonality elsewhere in the invasive range (northern Florida and California). Records of DRW occurrence in southern Florida were provided by the Florida Department of Agriculture and Consumer Services (FDACS). We focused our analysis on a welldocumented invasion near Homestead, Florida. The spatial scale of this invasion is relatively small $(\sim 250$ $\mathrm{km}^{2}$ ) and we expected that spread dynamics at this scale would represent natural, local dispersal and not humanmediated transport. The DRW was first recorded in this area in 1992 and new records of weevil occurrence, including date and location, were documented through 2006. Occurrence records came from targeted FDACS inspections of known host plants, citrus groves, and other agricultural production areas (C. Riherd and M. Thomas, FDACS, personal communication). We georeferenced the DRW occurrence records in ArcGIS and, 
for each year (1992-2006), estimated invaded area as the area of the minimum polygon that included all sites invaded in that year. We analyzed the spatial data at an annual time step because there were generally too few records per year to warrant finer temporal resolution. We applied the rule that, once invaded, a site remained invaded; this is consistent with field observations (M. Thomas, personal communication).

The invasion speed in one dimension also describes the rate of radial expansion in two dimensions, assuming that spread dynamics approximate an expanding circle (van den Bosch et al. 1992, Lensink 1997). The DRW invasion data were reasonably consistent with this assumption (Results), so we examined the radius invaded $\left(\sqrt{\mathrm{km}^{2} / \pi}\right)$ vs. time (years). The slope of this relationship gives the annual rate of radial expansion, which we estimated from the spatial data using maximum likelihood methods (Hilborn and Mangel 1997). Deviation between observed and predicted rates of spread could be the result of process error (e.g., fluctuation in parameters) or observation error (e.g., weevils occurred at a site but were not detected). We therefore generated two point estimates and 95\% confidence intervals for the speed of DRW invasion, assuming either source of error (Hilborn and Mangel 1997).

The DRW occurrence records probably do not capture the very early invasion dynamics, and the temporal resolution of the data set is much coarser than the timescale of predicted transient dynamics (years vs. weeks). For these reasons, the spatial spread data were not sufficient for the validation of transient dynamics, and we focus our comparison on observed and predicted asymptotic invasion speed. We scaled the predicted asymptotic speed from a weekly to an annual interval for consistency with the observed invasion speed $(\mathrm{km} /$ yr). This assumes that parameter values are constant throughout the year (i.e., no seasonality).

Because the predicted invasion speed was significantly greater than the observed speed (Results), we examined how much lower dispersal distances would need to be in order for the predicted invasion speed to fall within the confidence limits of observed invasion speed. We conducted this analysis using three sets of demographic parameter values from the empirically derived ranges (Table 1): bounds of the uncertainty ranges resulting in maximum local population growth rate $(\lambda=1.54)$, midpoints of uncertainty ranges $(\lambda=1.44)$, and bounds of the uncertainty ranges resulting in minimum local population growth rate $(\lambda=1.33)$.

\section{Results AND Discussion}

Mathematical models that couple demography and dispersal are potentially valuable tools for predicting the spatial spread of invasive exotic species and for developing strategies to slow or halt the spread of invaders (Fagan et al. 2002, Jongejans et al. 2008). Yet, the data on which these models are based are subject to high degrees of uncertainty, especially the dispersal component. Formulating management strategies in the face of uncertainty is among the most pressing issues in applied ecology. The Diaprepes root weevil (DRW) case study demonstrates how insights into the relative importance of demography and dispersal parameters underlying spread can be achieved even if parameter values are not perfectly known. Ours is not the first study to examine spatial spread dynamics under parameter uncertainty (e.g., Buckley et al. 2005, Tinker et al. 2008). However, our study advances this field by providing a critical assessment of local vs. global perturbation analyses, by examining spatial dynamics during both the transient and asymptotic phases of invasion, and by quantitatively comparing predicted and observed patterns of spatial spread.

\section{Asymptotic invasion dynamics and comparison of global vs. local perturbation analyses}

Simulations of the DRW invasion model provided estimates for rates of spatial spread under parameter uncertainty. The simulated estimates of asymptotic invasion speed were highly correlated with the analytical wave speed solutions (Pearson's product-moment correlation coefficient $=0.913, P<0.0001)$. This result gives us confidence that our numerical simulations accurately captured the dynamics of Eq. 1. The mean asymptotic rate of spatial spread, accounting for uncertainty in the demography and dispersal data, was $0.024 \mathrm{~km} /$ week $(95 \% \mathrm{CI}=0.02-0.028 \mathrm{~km} /$ week $)$.

We used partial rank correlation coefficients (PRCC's) to estimate the relative contributions of each parameter to rates of spatial spread. This global perturbation analysis, based on resampling values of all parameters from empirically derived uncertainty ranges, clearly identified the mean dispersal distances of pre-reproductive and mature adult weevils $\left(\alpha_{\mathrm{J}}\right.$ and $\alpha_{\mathrm{A}}$, respectively) as the parameters having the greatest effects on the asymptotic invasion speed; dispersal by mature adults had a greater effect than dispersal by prereproductive adults (Table 2). Two additional parameters, early-instar larval survival $\left(\sigma_{\mathrm{N}}\right)$ and development time $\left(\tau_{N}\right)$, were in the 75 th percentile of relative importance (absolute value of PRCC).

For comparison, we asked how well lower-level elasticities (LLE), the more traditional, local perturbation approach, could have predicted the parameter contributions that we detected with the global PRCC analysis. We found mixed results (Table 2). First, LLEs were consistent in sign with PRCC results; development times had negative values for both perturbation metrics because increasing their values decreased the rate of local population growth, which retarded propagule production and, hence, spatial spread. In addition, the LLEs correctly identified the dispersal parameters as having major effects on the asymptotic invasion speed. However, the LLEs indicated that pre-reproductive dispersal had a greater impact on spread than dispersal 
by mature adults, which is inconsistent with the simulation results. Furthermore, the local sensitivity analysis identified as important (within the 75th percentile of elasticity value) two demographic parameters (late-instar larva survival, $\sigma_{\mathrm{L}}$, and pre-reproductive adult survival, $\sigma_{\mathrm{J}}$ ) that had weak effects in the simulated invasions, and failed to identify two other demographic parameters (early-instar larva development time, $\tau_{\mathrm{N}}$, and survival, $\sigma_{\mathrm{N}}$ ) that had relatively large effects in the simulated invasions (Table 2). Thus, over the range of uncertainty present in the data, local sensitivity analysis provided unreliable predictions for the effects of demography and dispersal parameters on the velocity of weevil invasions. These results support previous suggestions (Hodgson and Townley 2004, Tenhumberg et al. 2008) that local perturbation analyses may give misleading results when parameter values are poorly known, as is often the case in applied ecology. We therefore suggest that global methods of perturbation analyses, such as those presented here, find wider application, particularly in studies of spatial spread, because dispersal parameters are prone to high uncertainty.

\section{Parameter contributions to asymptotic vs. transient peak invasion speed}

The role of human-mediated transport in initiating new invasions of this and other exotic pest species motivated our interests in transient spatial dynamics. Biological invasions that start in a single location with a single life stage, as many human-mediated invasions likely do, could exhibit strong transient dynamics because they deviate from both the equilibrium wave shape and the equilibrium stage structure. We found that DRW invasions that began with the introduction of mature adults reached a transient peak invasion speed of $0.037(0.03-0.045) \mathrm{km} /$ week (mean and 95\% CI), significantly greater than the asymptotic rate of spread. Such short-term bursts of spatial spread during the transient phase can have lasting effects on patterns of range expansion and may result in a greater area invaded than expected based solely on asymptotic predictions (Fig. 1). In addition, the extent of range expansion achieved early in an invasion may determine the importance of Allee effects (Kot et al. 1996) and the efficacy of biological control agents in slowing the spread of invaders (Fagan et al. 2005). For these reasons, it is important to know if management strategies for reducing asymptotic invasion speed are also effective in reducing transient peaks.

We examined the consistency of parameter contributions between asymptotic and transient peak DRW invasion speeds. Fig. 3 plots the partial rank correlation coefficients for transient vs. asymptotic speed, where parameters that fall on the 1:1 line contribute equally to both phases of spread. We found that the dispersal parameters $\left(\alpha_{\mathrm{J}}\right.$ and $\left.\alpha_{\mathrm{A}}\right)$, which had the greatest effects on asymptotic speed, also had the greatest effects on
TABLE 2. Partial rank correlation coefficients (PRCC) and lower-level elasticities (LLE) for effects of demography and dispersal parameters on the asymptotic invasion speed.

\begin{tabular}{lcc}
\hline \hline Parameter & PRCC ("global") & LLE ("local") \\
\hline Stage duration & & \\
$\tau_{\mathrm{E}}$ & -0.16 & -0.078 \\
$\tau_{\mathrm{N}}$ & $\mathbf{- 0 . 2}$ & -0.082 \\
$\tau_{\mathrm{L}}$ & -0.16 & -0.12 \\
$\tau_{\mathrm{P}}$ & -0.062 & -0.098 \\
$\tau_{\mathrm{J}}$ & -0.17 & -0.14 \\
$\tau_{\mathrm{A}}$ & 0.13 & 0.032 \\
Survival & & \\
$\sigma_{\mathrm{E}}$ & 0.19 & 0.27 \\
$\sigma_{\mathrm{N}}$ & $\mathbf{0 . 2 9}$ & 0.17 \\
$\sigma_{\mathrm{L}}$ & 0.014 & $\mathbf{0 . 2 8}$ \\
$\sigma_{\mathrm{P}}$ & 0.031 & 0.23 \\
$\sigma_{\mathrm{J}}$ & 0.14 & $\mathbf{0 . 4 0}$ \\
Fecundity, $\phi$ & 0.065 & 0.22 \\
Dispersal & & \\
$\boldsymbol{\alpha}_{\mathrm{A}}$ & & $\mathbf{0 . 3}$ \\
$\boldsymbol{\alpha}_{\mathrm{J}}$ & $\mathbf{0 . 9}$ & $\mathbf{0 . 7}$ \\
\hline
\end{tabular}

Note: Bolded values show correlation coefficients or elasticities in 75 th percentile of each respective data set.

transient speed. The demographic parameter with the greatest effect on transient wave speed was $\tau_{\mathrm{J}}$, the duration of the pre-reproductive adult stage (Table 2). Interestingly, this parameter had a positive effect on transient wave speed but a strong negative effect on asymptotic speed. We observe that $\tau_{\mathbf{J}}$ has a positive effect on stasis of pre-reproductive adults (element 5,5 of the transition matrix, $\mathbf{A}_{5,5}$ ), but negative effects on graduation rate $\left(\mathbf{A}_{6,5}\right)$ and fecundity of graduating females $\left(\mathbf{A}_{1,5}\right)$ (see sensitivity formulae in the Appendix). During transient (but not asymptotic) dynamics, the positive effects of pre-reproductive development time on stasis, coupled with the movement potential of pre-reproductive adults, outweighed the negative contributions of this parameter via alternate demographic pathways. Generally, though, demographic parameters contributed weakly to transient speed (PRCCs are clustered near zero on the transient axis; Fig. 3), regardless of whether they had positive, negative, or no effects on asymptotic speed. Thus, transient spatial spread, even more so than asymptotic spread, was dominated by how far the farthest traveling individuals move.

\section{Implications of perturbation results for DRW management}

Strong effects of dispersal parameters on invasion speed are a highly consistent result across taxa and modeling approaches (e.g., Kot et al. 1996, Neubert and Caswell 2000, Buckley et al. 2005, Jacquemyn et al. 2005, Shea and Skarpaas 2007). This is rather bad news from a management perspective because manipulating movement behavior is exceedingly difficult. The best that invasive species managers can hope for is to 


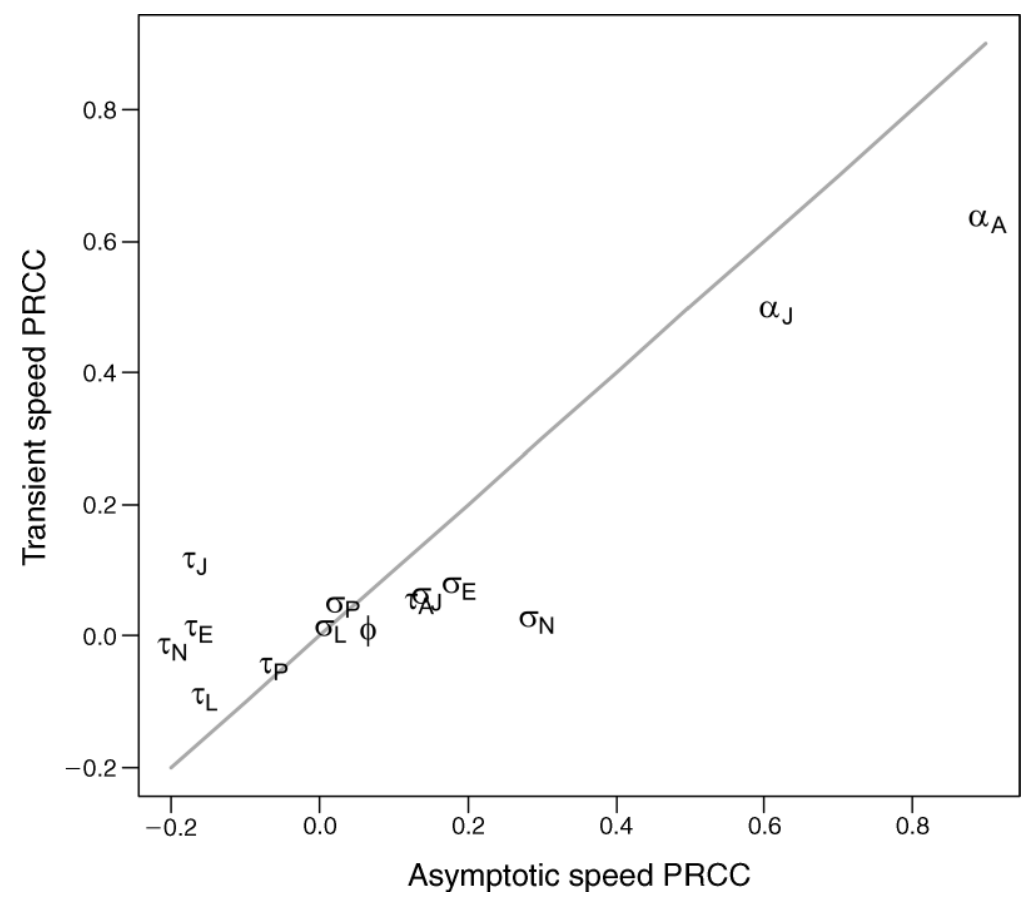

FIG. 3. Comparison of parameter effects (PRCC, partial rank correlation coefficients) on transient and asymptotic invasion speeds, estimated from the global perturbation analysis. Parameters for development time $(\tau)$, survival $(\sigma)$, fecundity $(\phi)$, and dispersal $(\alpha)$ are identified by age class (E, egg; N, early-instar larva; L, late-instar larva; P, pupa, J, pre-reproductive adult; A, adult); see Tables 1 and 2. Parameters that fall on the 1:1 line (in gray) contribute equally to both components of spatial spread.

manipulate local demography such that local population growth, and hence the number of propagules available for dispersal, is reduced. However, in the DRW case and in general, this will never be as effective in slowing spread as manipulating the probability distribution that governs how far those propagules move.

Biological control is one common approach to manipulating the demographic parameters of pest insects. Numerous natural enemies have been investigated or released for the biological control of DRW, including egg parasitoids (Hall et al. 2001), entomopathogenic nematodes that attack larvae (McCoy et al. 2000), and an entomopathogenic fungus that kills adults (Grafton-Cardwell et al. 2004). Results from our perturbation analysis indicate that, of the demographic pathways that are potential targets of biological control (i.e., survival parameters), reductions in early-instar larval survival would have the greatest effect on asymptotic invasion speed (Table 2). Entomopathogenic nematodes, the larval enemies, may therefore offer the greatest potential for reducing natural rates of spatial spread, although predation on late-instar larvae would be less effective in reducing spread than predation on early-instar larvae (Table 2). Shapiro et al. (1999) found that early DRW larval instars were more susceptible to nematode infection than late instars, a feature that could enhance the effects of nematodes on invasion speed. Despite this potential, the large-scale spatial dynamics of the DRW invasion in Florida, as well as its introduction to Texas and California, are thought to be dominated by human-mediated movement of infested plant material, with natural dispersal playing a relatively small role (Bas et al. 2000). While local management might effectively reduce local spread, these efforts must be combined with efforts to prevent "inflation" of the dispersal kernel caused by human commerce.

Incipient invasions resulting from human-mediated transport may exhibit transient spread dynamics that strongly deviate from long-term patterns. Unfortunately, we found that transient DRW invasion speeds were generally unresponsive to variation in demographic parameters, the potential targets of management. Thus, effective strategies for decelerating asymptotic invasion speed (biological control of larvae, for example) may have little effect in dampening the transient peak, at least for invasions that begin with mature adults; alternative initial conditions may yield qualitatively different transient dynamics. Of course, any intervention during the transient phase would require a low detection threshold and relatively fast management action. Reduction of transient peak invasion speed may therefore be more realistic for organisms with seasonal reproduction, such as plants, where nonequilibrium dynamics can play out over multiple years.

\section{Comparison of predicted and observed spread dynamics}

While the use of spatial models for understanding and predicting invasions is increasingly popular, relatively 

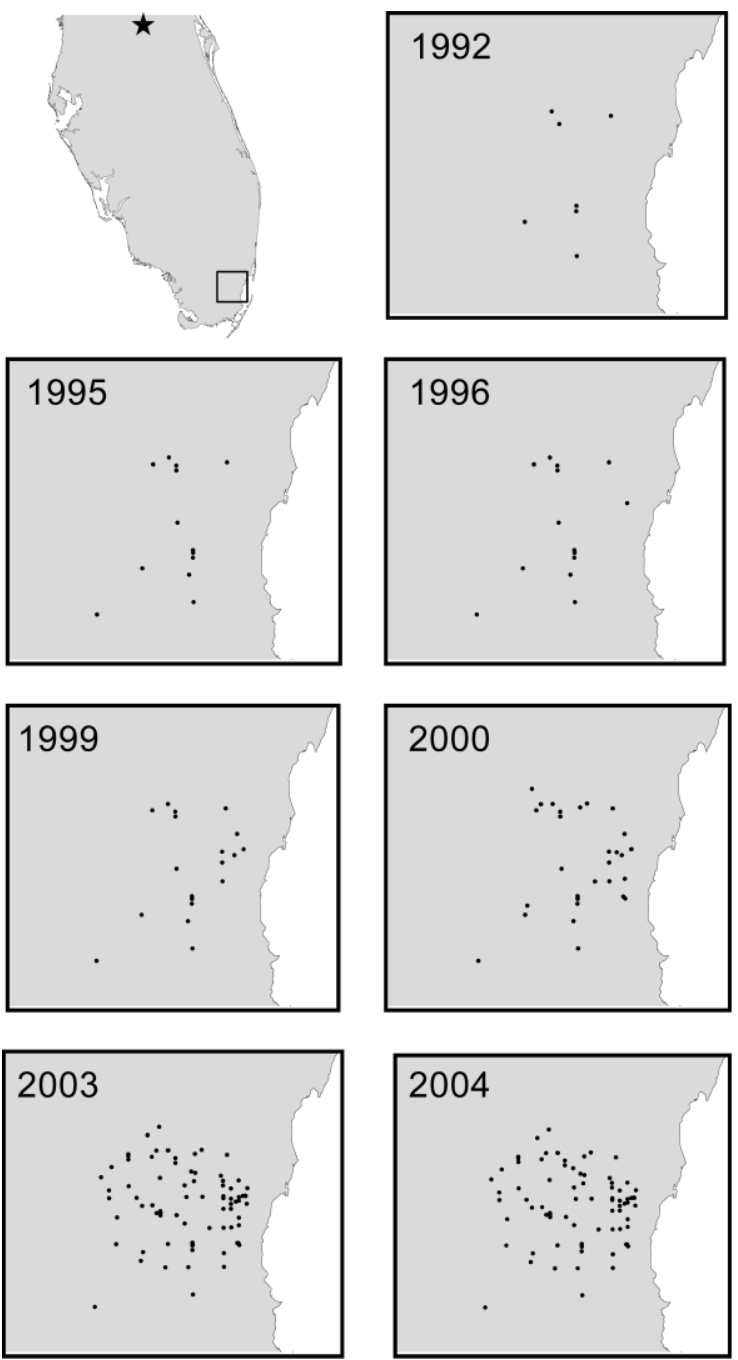
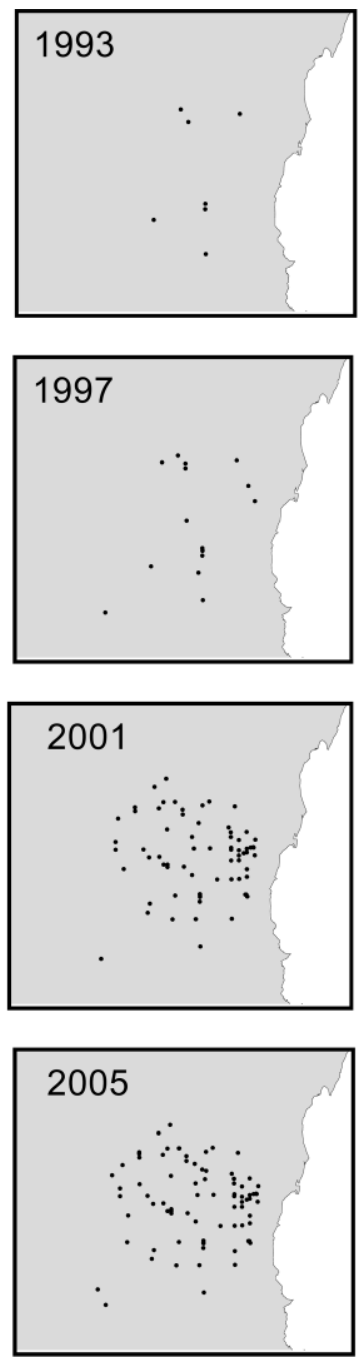
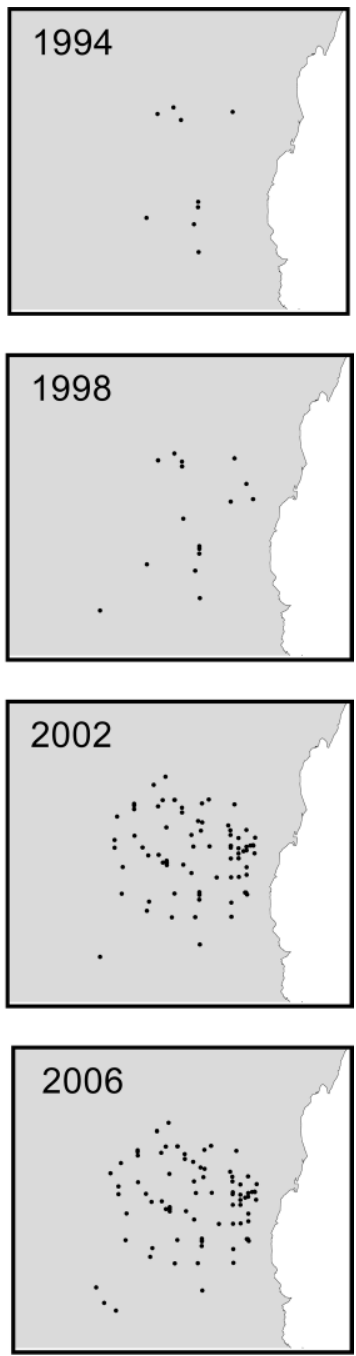

FIG. 4. Time series of DRW invasion near Homestead, Florida, USA. Occurrence records were provided by the Florida Department of Agriculture and Consumer Services. The star in the location map denotes Apopka, Florida, where the weevil was first detected in 1964. The rectangle indicates the focal area shown over time (1992-2006) in other panels.

few studies have compared model predictions with independent estimates of range expansion (Andow et al. 1990, Grosholz 1996, Veit and Lewis 1996, Tinker et al. 2008). Records of DRW range expansion in southern Florida, collected by a state management agency (FDACS), provided the opportunity to compare predictions from our model with observed spatial spread dynamics. The time series of invasive range expansion (1992-2006) is mapped in Fig. 4. The spatial extent of the invasion near Homestead, Florida increased over the observation period (Fig. 5A). The fitted slope of the invasion radius vs. time, assuming observation error, was $0.296 \mathrm{~km} / \mathrm{yr}(95 \% \mathrm{CI}=0.271-0.321 \mathrm{~km} / \mathrm{yr})$. When the slope was fitted under process error, the point estimate was similar $(0.253 \mathrm{~km} / \mathrm{yr})$ although the $95 \% \mathrm{CI}$ was larger $(0.0724-0.434 \mathrm{~km} / \mathrm{yr})$, reflecting the temporal heterogeneity in radial expansion (Fig. 5A). When we compared predicted and observed rates of spread, we found that the asymptotic invasion speed predicted from the demography and dispersal data was significantly greater than invasion speeds estimated from the DRW occurrence records (Fig. 5B). Mismatch between predicted and observed invasion dynamics is a common result (Grosholz 1996). We will discuss possible causes of this discrepancy for the DRW.

First, given the importance of pre-reproductive and mature adult dispersal distances $\left(\alpha_{\mathrm{J}}\right.$ and $\left.\alpha_{\mathrm{A}}\right)$ as determinants of invasion speed, we asked: By how much would these parameter values need to be reduced in order for the predicted invasion speed to fall within the confidence limits of observed invasion speed (estimated under process error)? Within the empirically derived uncertainty ranges of the demographic parameters, we found that reductions of $42-54 \%$ for $\alpha_{\mathrm{J}}$ and $50-61 \%$ for 

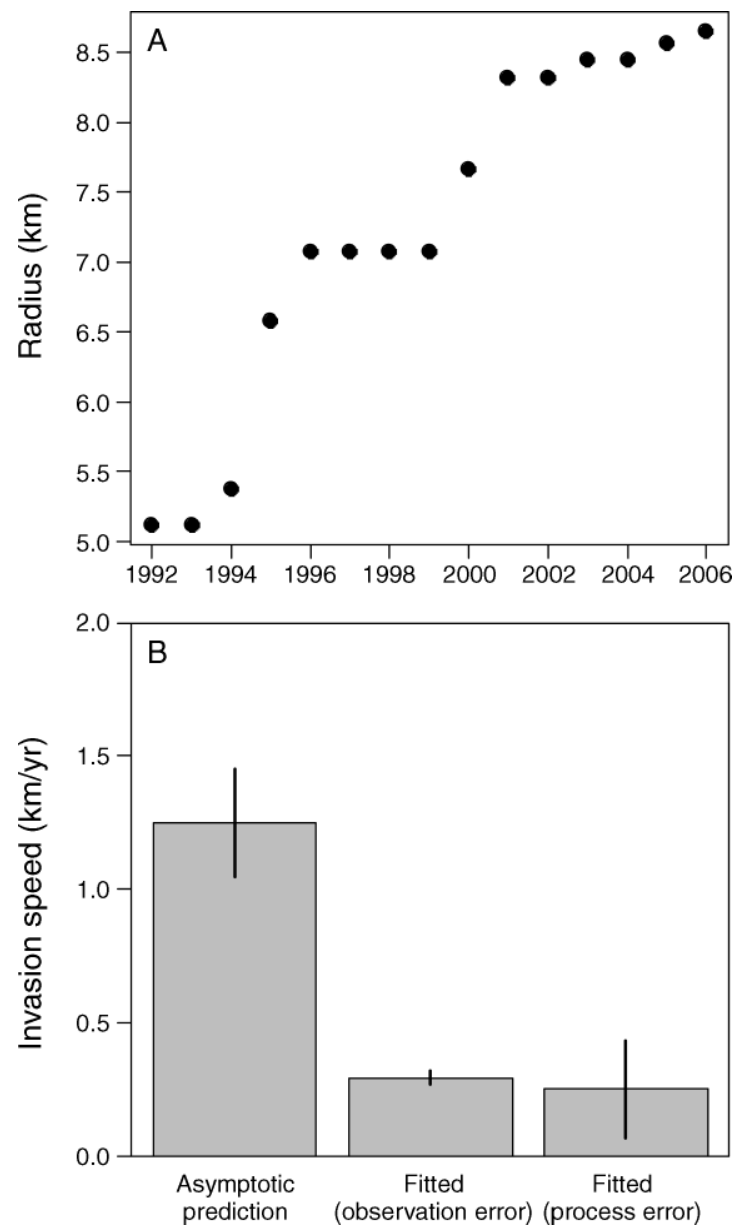

FIG. 5. (A) Observed change in the radius of the DRW invasion through time, estimated from the time series of DRW occurrence records, 1992-2006 (Fig. 4). (B) Comparison of predicted and observed rates of DRW range expansion. The asymptotic prediction was based on the demography and dispersal data, and the observed values were fitted to the data in panel (A), assuming either observation or process error. Error bars show $95 \%$ confidence intervals.

$\alpha_{\mathrm{A}}$ would be necessary, in combination (Fig. 6). Thus, one possible explanation for the over-prediction of DRW invasion speed is that the DRW dispersal experiments (Fig. 2) provided overestimates of dispersal parameters, perhaps due to density dependence in movement behavior. In these experiments, marked individuals were released in large aggregates, probably at higher densities than those at which natural dispersal would occur. If individual movement probabilities increase with local density, then this common experimental approach could overestimate dispersal distances. However, we think it unlikely that the experiments of Nigg et al. (2001) overestimated dispersal on the order necessary to account for the mismatch between predicted and observed invasion speeds. Mark-release-resight experiments are more likely to underestimate than overestimate dispersal distances due to the low proba- bility of detecting rare, long-distance movement (Nathan et al. 2003).

We note that the reduction in $\alpha_{\mathrm{J}}$ and $\alpha_{\mathrm{A}}$ required to move the predicted invasion speed closer to observations decreased as values for the demographic parameters became increasingly "pessimistic" (lower survival rates and longer development times; Fig. 6). Thus, a second explanation (not exclusive with the first) is that the demographic parameter values used to generate predictions (Table 1) were unrealistically favorable. We think this is a plausible explanation. The survival and development time data were collected under laboratory conditions, and therefore did not account for any effects of natural enemies, including intentionally released biological control agents. Agents released to date would likely reduce egg survival (parasitic wasps) and earlyinstar larval survival (entomopathogenic nematodes), two demographic parameters with relatively large effects on asymptotic invasion speed (Fig. 3). Also, we used demographic parameter estimates from temperatures corresponding to the mean annual air temperature in the region. Temperatures in the soil are probably cooler, which could increase the development times of early- and late-instar larvae (Lapointe 2000) and further retard the rate of spread (Fig. 3).

Additionally, we recognize spatial and temporal variability in parameter values, including seasonality,

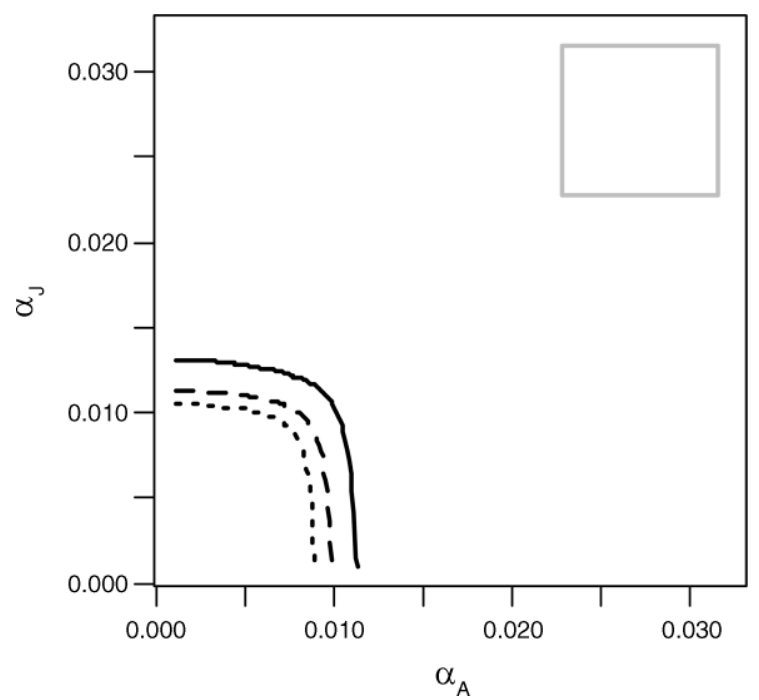

FIG. 6. Joint parameter space of mature adult $\left(\alpha_{A}\right)$ and prereproductive adult $\left(\alpha_{\mathrm{J}}\right)$ mean dispersal distance. Black lines indicate the parameter value combinations for which the predicted invasion speed (km/week) equals the upper $95 \%$ confidence limit of observed invasion speed (estimated under process error; Fig. 5). Line styles correspond to three sets of demographic parameter values (Table 1): solid, "pessimistic" bounds of uncertainty ranges; dashed, midpoints of uncertainty ranges; dotted, "optimistic" bounds of uncertainty ranges. The gray box indicates the joint uncertainty range of dispersal parameters, estimated from release-recapture experiments (Fig. 2), used to generate predicted invasion speed. 
as a third, related explanation for the mismatch between predicted and observed invasion speeds. That is, our empirical estimates for demography and dispersal parameters may have been reasonable during certain times of year or in certain places, but not others. Like most demographic studies, we assumed that parameter values were constant, and therefore focused our comparison of predicted and observed invasion dynamics on a relatively small region in southern Florida, where seasonality is minimal. However, this area experiences an average of 10-15 days per year when air temperatures are lower than the threshold for female oviposition $\left(15^{\circ} \mathrm{C}\right)$, and $4-5$ days per year when soil temperatures fall below the threshold for larval development $\left(12^{\circ} \mathrm{C}\right.$; Lapointe et al. 2007). Also, although adult weevils occur yearround in southern Florida, nothing is known regarding seasonality of adult movement behavior. If adults are relatively sedentary during certain times of year, this would retard annual rates of spread relative to our predictions. Models that account for environmental influences on demographic and dispersal processes may be necessary for more accurate predictions of DRW spatial dynamics, particularly in parts of the invasive range with stronger seasonality (northern Florida and California). For this reason, a discrete-space, statistical model (e.g., Havel et al. 2002, Gilbert et al. 2005) might provide more accurate predictions than our mechanistic, population dynamics model. Statistical invasion models can generate predictions of spread by characterizing correlations between environmental variables and presence-absence of the invader. Studies that quantitatively compare the predictive accuracy of these alternative approaches would be valuable. However, because statistical models do not explicitly consider underlying mechanisms, they are not appropriate for estimating the contributions of demographic rates and dispersal processes toward spread dynamics, the primary objective of our study.

Finally, we note that DRW radial expansion in southern Florida was not exactly linear with respect to time (Fig. 5). Rather, periods of spread (1993-1996, 1999-2001) were interspersed with periods of relative stasis, a pattern observed in other invasive insects (e.g., Johnson et al. 2006). When we restricted analysis to periods of expansion, invasion speed estimates were much closer to the model prediction and, for the period 1993-1996, confidence intervals of predicted and observed invasion speeds overlapped. Temporal heterogeneity in invasion speed may be driven by endogenous factors such as Allee effects (Johnson et al. 2006) or may reflect human activities such as use of insecticide or biological control, which often go undocumented. Identifying sources of temporal and spatial variation in rates of range expansion would be useful for verifying and refining theory for population spatial spread (Sawyer et al. 2007, Urban et al. 2008).

\section{Conclusions}

With this case study, we have shown that models of stage-structured invasions can provide valuable insights into rates and mechanisms of spread, even if key parameters are poorly known. However, when parameter uncertainty is large, as is often the case, global perturbation analyses are needed to accurately identify which points in the life cycle should be targets of management. This study also demonstrates that effective strategies for reducing spread during the asymptotic phase may have little effect during the transient phase. Because transient peaks in invasion speed can influence long-term patterns of range expansion, greater attention to nonequilibrium spatial dynamics is warranted. Finally, contact between invasion theory and application would be facilitated by more quantitative comparisons of predicted and observed spread dynamics and by investigations into sources of mismatch.

\section{ACKNOWLEDGments}

We thank Brian Inouye and two anonymous reviewers for valuable comments on this manuscript. Michael Thomas and colleagues at the Florida Department of Agriculture and Consumer Services generously provided DRW occurrence records and other useful information. This work was supported by a USDA National Research Initiative postdoctoral fellowship (2007-02270) to T. Miller and the University of Nebraska Research Council Layman Funds to B. Tenhumberg.

\section{Literature Cited}

Andow, D. A., P. Kareiva, S. Levin, and A. Okubo. 1990. Spread of invading organisms. Landscape Ecology 4:177188.

Bas, B., Z. Dalkilic, T. L. Peever, H. N. Nigg, S. E. Simpson, F. G. Gmitter, Jr., and R. C. Adair. 2000. Genetic relationships among Florida Diaprepes abbreviatus (Coleoptera: Curculionidae) populations. Environmental Entomology 93:459-467.

Beavers, J. B. 1982. Biology of Diaprepes abbreviatus (Coleoptera: Curculionidae) reared on an artificial diet. Florida Entomologist 65:263-270.

Beavers, J. B., and A. G. Selhime. 1976. Population dynamics of Diaprepes abbreviatus in an isolated citrus grove in central Florida. Journal of Economic Entomology 69:9-10.

Blower, S. M., and H. Dowlatabadi. 1994. Sensitivity and uncertainty analysis of complex models of disease transmission: an HIV model as an example. International Statistical Review 62:229-243.

Buckley, Y. M., E. Brockerhoff, L. Langer, N. Ledgard, H. North, and M. Rees. 2005. Slowing down a pine invasion despite uncertainty in demography and dispersal. Journal of Applied Ecology 42:1020-1030.

Burnham, K. P., and D. R. Anderson. 2002. Model selection and multimodel inference: a practical information-theoretic approach. Second edition. Springer, New York, New York, USA.

Caswell, H. 2001. Matrix population models. Sinauer, Sunderland, Massachusetts, USA.

Caswell, H. 2007. Sensitivity analysis of transient population dynamics. Ecology Letters 10:1-15.

Conover, W. J. 1980. Practical nonparametric statistics. Second edition. John Wiley, New York, New York, USA.

Elton, C. S. 1958. The ecology of invasions by animals and plants. Methuen, London, UK. 
Fagan, W. F., M. A. Lewis, M. G. Neubert, C. Aumann, J. L. Apple, and J. G. Bishop. 2005. When can herbivores slow or reverse the spread of an invading plant? A test case from Mount St. Helens. American Naturalist 166:669-685.

Fagan, W. F., M. A. Lewis, M. G. Neubert, and P. van den Driessche. 2002. Invasion theory and biological control. Ecology Letters 5:148-157.

Gilbert, M., S. Guichard, J. Freise, J.-C. Grégoire, W. Heitland, N. Straw, C. Tilbury, and S. Augustin. 2005. Forecasting Cameraria ohridella invasion dynamics in recently invaded countries: from validation to prediction. Journal of Applied Ecology 42:805-813.

Grafton-Cardwell, E. E., K. E. Godfrey, J. E. Pena, C. W. McCoy, and R. F. Luck. 2004. Diaprepes root weevil. University of California Division of Agriculture and Natural Resources Publication 8131. University of California-Davis, Oakland, California, USA.

Grosholz, E. D. 1996. Contrasting rates of spread for introduced species in terrestrial and marine environments. Ecology 77:1680-1686.

Hall, D. G. 2001. History and importance of Diaprepes to Florida. In Proceedings, Diaprepes short course. University of Florida Institute of Food and Agricultural Sciences, Gainesville, Florida, USA.

Hall, D. G., J. Pena, R. Franqui, R. Nguyen, P. Stansly, C. W. McCoy, S. L. LaPointe, R. C. Adair, and B. Bullok. 2001. Status of biological control by egg parasitoids of Diaprepes abbreviatus (Coleoptera: Curculionidae) in citrus in Florida and Puerto Rico. BioControl 46:61-70.

Hastings, A., et al. 2005. The spatial spread of invasions: new developments in theory and evidence. Ecology Letters 8:91101.

Havel, J. E., J. B. Shurin, and J. R. Jones. 2002. Estimating dispersal from patterns of spread: spatial and local control of lake invasions. Ecology 83:3306-3318.

Hilborn, R., and M. Mangel. 1997. The ecological detective: confronting models with data. Princeton University Press, Princeton, New Jersey, USA.

Hodgson, J., and S. Townley. 2004. Linking management changes to population dynamic responses: the transfer function of a projection matrix. Journal of Applied Ecology 41:1155-1161.

Horvitz, C. C., and D. W. Schemske. 1995. Spatiotemporal variation in demographic transitions of a tropical understory herb: projection matrix analysis. Ecological Monographs 65: 155-192.

Jacquemyn, H., R. Brys, and M. G. Neubert. 2005. Fire increases invasive spread of Molina caerulea mainly through changes in demographic parameters. Ecological Applications 15:2097-2108.

Johnson, D. M., A. M. Liebhold, P. C. Tobin, and O. N. Bjornstad. 2006. Allee effects and pulsed invasion by the gypsy moth. Nature 444:361-363.

Jongejans, E., O. Skarpaas, and K. Shea. 2008. Dispersal, demography, and spatial population models for conservation and control management. Perspectives in Plant Ecology, Evolution, and Systematics 9:153-170.

Koons, D. N., J. B. Grand, B. Zinner, and R. F. Rockwell. 2005. Transient population dynamics: relations to life history and initial population state. Ecological Modelling 185:283-297.

Kot, M., M. A. Lewis, and P. van den Driessche. 1996. Dispersal data and the spread of invading organisms. Ecology 77:2027-2042.

Lapointe, S. L. 2000. Thermal requirements for development of Diaprepes abbreviatus (Coleoptera: Curculionidae). Environmental Entomology 29:150-156.

Lapointe, S. L. 2001. Effect of temperature on egg development of Diaprepes abbreviatus (Coleoptera: Curculionidae). Florida Entomologist 84:298-299.
Lapointe, S. L., D. M. Borchert, and D. G. Hall. 2007. Effect of low temperatures on mortality and oviposition in conjunction with climate mapping to predict spread of the root weevil Diaprepes abbreviatus and introduced natural enemies. Environmental Entomology 36:73-82.

Lensink, R. 1997. Range expansion of raptors in Britain and the Netherlands since the 1960's: testing an individual-based diffusion model. Journal of Animal Ecology 66:811-826.

McCoy, C. W., D. I. Shapiro, L. W. Duncan, and K. Nguyen. 2000. Entomopathogenic nematodes and other natural enemies as mortality factors for larvae of Diaprepes abbreviatus (Coleoptera: Curculionidae). Biological Control 19:182-190.

McMahon, S. M., and C. J. E. Metcalf. 2008. Transient sensitivities of non-indigenous shrub species indicate complicated invasion dynamics. Biological Invasions 10:833-846.

Nathan, R., G. Perry, J. T. Cronin, A. E. Strand, and M. L. Cain. 2003. Methods for estimating long-distance dispersal. Oikos 103:261-273.

National Research Council. 2002. Predicting invasions of nonindigenous plants and plant pests. National Academy Press, Washington, D.C., USA.

Neubert, M. G., and H. Caswell. 2000. Demography and dispersal: calculation and sensitivity analysis of invasion speed for structured populations. Ecology 81:1613-1628.

Nigg, H. N., S. E. Simpson, L. E. Ramos, T. Tomerlin, J. M. Harrison, and N. Cuyler. 2001. Distribution and movement of adult Diaprepes abbreviatus (Coleoptera: Curculionidae) in a Florida citrus grove. Florida Entomologist 84:641-651.

Okubo, A., and S. A. Levin. 2002. Diffusion and ecological problems: modern perspectives. Springer-Verlag, New York, New York, USA.

Ricker, W. E. 1954. Stock and recruitment. Journal of the Fisheries Research Board of Canada 11:559-623.

Sawyer, H., R. M. Nielson, F. G. Lindzey, L. Keith, J. H. Powell, and A. A. Abraham. 2007. Habitat selection of Rocky Mountain elk in a nonforested environment. Journal of Wildlife Management 71:868-874.

Shapiro, D. I., J. R. Cate, J. Pena, A. Hunsberger, and C. W. McCoy. 1999. Effects of temperature and host age on suppression of Diaprepes abbreviatus (Coleoptera: Curculionidae) by entomopathogenic nematodes. Journal of Economic Entomology 92:1086-1092.

Sharov, A. A., and A. M. Liebhold. 1998. Model of slowing the spread of gypsy moth (Lepidoptera: Lymantriidae) with a barrier zone. Ecological Applications 8:1170-1179.

Shea, K., and O. Skarpaas. 2007. Dispersal patterns, dispersal mechanisms, and invasion wave speeds for invasive thistles. American Naturalist 170:421-430.

Simpson, S. E., H. N. Nigg, N. C. Coile, and R. A. Adair. 1996. Diaprepes abbreviatus (Coleoptera: Curculionidae): host plant associations. Environmental Entomology 25:333-349.

Skaria, M., and J. V. French. 2001. Phytophthora disease of citrus associated with root weevils in Texas. Phytopathology 91:S203.

Tenhumberg, B., S. M. Louda, J. O. Eckberg, and M. Takahashi. 2008. Monte Carlo analysis of parameter uncertainty in matrix models for the weed Cirsium vulgare. Journal of Applied Ecology 45:438-447.

Tenhumberg, B., A. J. Tyre, and R. Rebarber. 2009. Model complexity affects transient population dynamics following a dispersal event: a case study with pea aphids. Ecology 90: 1878-1890.

Tinker, M. T., D. F. Doak, and J. A. Estes. 2008. Using demography and movement behavior to predict range expansion of the southern sea otter. Ecological Applications 18:1781-1794.

Townley, S., D. Carslake, O. Kellie-Smith, D. McCarthy, and D. Hodgson. 2007. Predicting transient amplification in 
perturbed ecological systems. Journal of Applied Ecology 44: 1243-1251.

Urban, M. C., B. L. Phillips, D. K. Skelly, and R. Shine. 2008. A toad more traveled: the heterogeneous invasion dynamics of cane toads in Australia. American Naturalist 171:E134E148.

van den Bosch, F., R. Hengeveld, and J. A. J. Metz. 1992. Analysing the velocity of animal range expansion. Journal of Biogeography 19:135-150.
Veit, R. R., and M. A. Lewis. 1996. Dispersal, population growth, and the Allee effect: dynamics of the house finch invasion of Eastern North America. American Naturalist 148:225-274

Woodruff, R. E. 1968. The present status of a West Indian weevil (Diaprepes abbreviatus (L.)) in Florida (Coleoptera: Curculionidae). Florida Department of Agriculture, Division of Plant Industry and Entomology. Publication 77. Florida Department of Agriculture, Gainesville, Florida, USA.

\section{APPENDIX}

Analytical formulas for the sensitivities of asymptotic invasion speed to lower-level demography and dispersal parameters (Ecological Archives A020-018-A1). 
Tom E. X. Miller and Brigitte Tenhumberg. 2010. Contributions of demography and dispersal parameters to the spatial spread of a stage-structured insect invasion. Ecological Applications 20:620-633.

Appendix A. Analytical formulae for the sensitivities of asymptotic invasion speed to lower-level demography and dispersal parameters.

TABLE A1. Analytical formulae for the sensitivities of asymptotic invasion speed to lower-level demography and dispersal parameters. Elasticities were estimated from the sensitivities as in Neubert and Caswell (2000). 
$\tau_{E}$

$$
\frac{\partial c^{*}}{\partial \tau_{E}}=\frac{\sigma_{E}}{\tau_{E}^{2}}\left(\frac{\partial c^{*}}{\partial a_{1,1}}\right)-\frac{\sigma_{E}}{\tau_{E}^{2}}\left(\frac{\partial c^{*}}{\partial a_{2,1}}\right)
$$

$\sigma_{E}$

$$
\frac{\partial c^{*}}{\partial \sigma_{E}}=\frac{\tau_{E}-1}{\tau_{E}}\left(\frac{\partial c^{*}}{\partial a_{1,1}}\right)+\frac{1}{\tau_{E}}\left(\frac{\partial c^{*}}{\partial a_{2,1}}\right)+\frac{\phi \tau_{J} \sigma_{J}}{4 \sqrt{\sigma_{E}}}\left(\frac{\partial c^{*}}{\partial a_{1,5}}\right)+\frac{\phi\left(1+2^{-\frac{1}{\tau_{A}}}\right)}{4 \sqrt{\sigma_{E}}}\left(\frac{\partial c^{*}}{\partial a_{1,6}}\right)
$$

$\tau_{N}$

$$
\frac{\partial c^{*}}{\partial \tau_{N}}=\frac{\sigma_{N}}{\tau_{N}^{2}}\left(\frac{\partial c^{*}}{\partial a_{2,2}}\right)-\frac{\sigma_{N}}{\tau_{N}{ }^{2}}\left(\frac{\partial c^{*}}{\partial a_{3,2}}\right)
$$

$\sigma_{N}$

$$
\frac{\partial c^{*}}{\partial \sigma_{N}}=\frac{\tau_{N}-1}{\tau_{N}}\left(\frac{\partial c^{*}}{\partial a_{2,2}}\right)+\frac{1}{\tau_{N}}\left(\frac{\partial c^{*}}{\partial a_{3,2}}\right)
$$

$\tau_{L}$

$$
\frac{\partial c^{*}}{\partial \tau_{L}}=\frac{\sigma_{L}}{\tau_{L}^{2}}\left(\frac{\partial c^{*}}{\partial a_{3,3}}\right)-\frac{\sigma_{L}}{\tau_{L}^{2}}\left(\frac{\partial c^{*}}{\partial a_{4,3}}\right)
$$

$\sigma_{L}$

$$
\frac{\partial c^{*}}{\partial \sigma_{L}}=\frac{\tau_{L}-1}{\tau_{L}}\left(\frac{\partial c *}{\partial a_{3,3}}\right)+\frac{1}{\tau_{L}}\left(\frac{\partial c^{*}}{\partial a_{4,3}}\right)
$$

$\tau_{P}$

$$
\frac{\partial c^{*}}{\partial \tau_{P}}=\frac{\sigma_{N}}{\tau_{N}{ }^{2}}\left(\frac{\partial c^{*}}{\partial a_{4,4}}\right)-\frac{\sigma_{N}}{\tau_{N}^{2}}\left(\frac{\partial c^{*}}{\partial a_{5,4}}\right)
$$

$\sigma_{P}$

$$
\frac{\partial c^{*}}{\partial \sigma_{P}}=\frac{\tau_{P}-1}{\tau_{P}}\left(\frac{\partial c^{*}}{\partial a_{4,4}}\right)+\frac{1}{\tau_{P}}\left(\frac{\partial c^{*}}{\partial a_{5,4}}\right)
$$

$\tau_{J}$

$$
\frac{\partial c^{*}}{\partial \tau_{J}}=\frac{\sigma_{J}}{\tau_{J}^{2}}\left(\frac{\partial c^{*}}{\partial a_{5,5}}\right)-\frac{\sigma_{J}}{\tau_{J}^{2}}\left(\frac{\partial c^{*}}{\partial a_{6,5}}\right)-\frac{\sqrt{\sigma_{E}} \phi \sigma_{J}}{2 \tau_{J}^{2}}\left(\frac{\partial c^{*}}{\partial a_{1,5}}\right)
$$

$\sigma_{\jmath}$

$$
\frac{\partial c^{*}}{\partial \sigma_{J}}=\frac{\tau_{J}-1}{\tau_{J}}\left(\frac{\partial c^{*}}{\partial a_{5,5}}\right)+\frac{1}{\tau_{J}}\left(\frac{\partial c^{*}}{\partial a_{6,5}}\right)+\frac{\sqrt{\sigma_{E}} \phi \tau_{J}}{2}\left(\frac{\partial c^{*}}{\partial a_{1,5}}\right)
$$

$\tau_{A}$

$$
\frac{\partial c^{*}}{\partial \tau_{A}}=\frac{2^{\frac{1}{\tau_{A}}} \ln (2)}{\tau_{A}{ }^{2}}\left(\frac{\partial c^{*}}{\partial a_{6,6}}\right)+\frac{2^{-\frac{\tau_{A}+1}{\tau_{A}}} \sqrt{\sigma_{E}} \phi \ln (2)}{\tau_{A}{ }^{2}}\left(\frac{\partial c^{*}}{\partial a_{1,6}}\right)
$$

$$
\begin{gathered}
\frac{\partial c^{*}}{\partial \phi}=\frac{\sqrt{\sigma_{E}} \tau_{J} \sigma_{J}}{2}\left(\frac{\partial c^{*}}{\partial a_{1,5}}\right)+\frac{\sqrt{\sigma_{E}}\left(1+2^{-\frac{1}{T_{A}}}\right)}{2}\left(\frac{\partial c^{*}}{\partial a_{1,6}}\right) \\
\frac{\partial c^{*}}{\partial \alpha_{J}}=\sum_{i, j} \frac{a_{i j}}{\hat{s} \rho_{1}} \frac{2 \alpha_{J} \hat{s}^{2}}{\left(1-\alpha_{J}{ }^{2} \hat{s}^{2}\right)^{2}} \frac{\partial \rho_{1}}{\partial h_{i j}}
\end{gathered}
$$




\section{LITERATURE CITED}

Neubert, M. G., and H. Caswell. 2000. Demography and dispersal: calculation and sensitivity analysis of invasion speed for structured populations. Ecology 81:1613-1628.

[Back to A020-018] 